\title{
A Novel Field Effect Photodiode to Control the Output Photocurrent and Fast Optical Switching
}

\author{
Foad Sharafi, Ali A. Orouji, Member, IEEE and Mohammad Soroosh
}

\begin{abstract}
This paper presents a novel device named Field Effect Photodiode (FEPD) to overcome the inherent drawbacks of PIN Photodiode (PIN-PD) and having an accurate control of the output photocurrent either applying the regular PIN-PD as a fast optical switch that provides a desire Ion/Ioff ratio for optical applications in the nanoscale regime. The proposed device combines a Metal Semiconductor Field Effect Transistor (MESFET) and a regular PIN-PD device that can convert the incident light with photon energy greater than the semiconductor's bandgap to the regulated photocurrent by changing the gates bias which mounted over the absorption region. Our work include additional models such as bandgap narrowing, Shockley-Read-Hall (SRH), Auger (AUGER), the dependence of the carrier mobility on the doping concentration, Lombardi mobility model (CVT), Fermi statistic dependence (FERMIDIRAC), and Lateral electric field-dependent mobility. To extract and illustrate the electrical and optical results of both the regular PIN-PD and the proposed FEPD in this work, we have used TCAD tools as a semiconductor simulator.
\end{abstract}

Index Terms - PIN Photodiode; Field Effect Photodiode; Absorption region; Photon energy; Internal Quantum Efficiency.

\section{Introduction}

Photodiodes are optoelectronic devices that can absorb light and penetrate the absorbing region regarding the photon energy greater than the semiconductor's bandgap energy, the excited electron from the valance band to the conduction band, the generating electron-hole pairs, and results to the output photocurrent $[1,2]$. For description this mechanism, we should seek some optical and physical phenomena; internal quantum efficiency, extrinsic absorption mediated by defect, absorption coefficient, photoelectric effect, inter sub-band absorption in quantum well heterostructures, wavelength, intrinsic inter-band absorption in semiconductors, the intensity of incident light, and other phenomena [3].

Regarding the limitation of conventional PIN photodiodes (PIN-PD) in some optical communication applications, we need devices that can work in high-speed switching and high output power [4]. The PIN-PD was one of the most popular devices Manuscript submitted July 29, 2021.

F. Sharafi is with the Department of Electrical Engineering, Semnan University, Semnan, Iran (e-mail: f.sharafi@ semnan.ac.ir).

Ali A. Orouji is with the Department of Electrical Engineering, Semnan University, Semnan, Iran (e-mail: aliaorouji@ semnan.ac.ir).

M. Soroosh is with the Department of Electrical Engineering, Faculty of Engineering, Shahid Chamran University of Ahvaz, Ahvaz, Iran (e-mail: m.soroosh@scu.ac.ir). before the invention of the uni-traveling carrier photodiode devices (UTC-PD) [5,6]. It is used for the fiber-optic communications $[7,8]$ since it has a broad bandwidth of $67 \mathrm{GHz}$ and over $100 \mathrm{GHz}$. To explain the leak in the regular PIN-PD, we can mention the fact that since both electrons and holes are active carriers and the holes drift at a lower velocity, the main performance of the PIN-PD, such as bandwidth, effected by the velocity of the hole's drift $[9,10]$. Thus, it results in an inevitable trade-off between output power and other characteristics [11].

This paper proposes a novel device named Field Effect Photodiode (FEPD) to overcome the weakness of PIN-PD and accurate control of the output photocurrent either applying the regular PIN-PD as a fast optical switch that provides a desire $\mathrm{I}_{\mathrm{ON}} / \mathrm{I}_{\mathrm{OFF}}$ ratio for optical applications in the nanoscale regime. The proposed device combines a Metal Semiconductor Field Effect Transistor (MESFET) and a regular PIN-PD structure $[12,13]$. This paper introduces a novel device with a very simple structure and doping concentration that makes it suitable for fabrication by the standard process technology of the regular PIN photodiode and conventional MESFET structures despite two gates over the absorption region for the output current control $[14,15]$.

\section{FEPD structure and Modeling}

The proposed device structure in this work is generally based on self-aligned $\mathrm{n}-\mathrm{GaAs}$ as an absorption region material with indium tin oxide (ITO) as Schottky metal gate electrodes. When the surface of the device is illuminated by optical radiation between intervals from $300 \mathrm{~nm}$ to $800 \mathrm{~nm}$ from an external source, it couples into the device through the transparent ITO metal contact as a Schottky-gate electrode to modulate the electrical and frequency characteristics of the device. Due to some limitations such as increasing the recombination at the device's surface that leads to decreasing the absorption distance at shorter wavelengths and increasing dark current, tends to use of absorbing materials with bandgaps that are only modestly smaller than in comparison with the energy of the incident photons for obtaining the best efficiency and lower noise. To reach better results of the designed device, it should be noted that the detector designs, specifically engineered to ensure from the drift-dominated transport mechanism away from the illuminated surface area, have been demonstrated to improve the short-wavelength performance [16]. For the combination of the mentioned factors; interested to the use of hetero-junction structure such GaAs/AlGaAs for high-speed detectors systems 
since mole fractions of $\mathrm{Al}_{\mathrm{x}} \mathrm{Ga}_{1-\mathrm{x}} \mathrm{As}$ provide a good match to the required bandgap and $\mathrm{GaAs} / \mathrm{AlGaAs}$ hetero-junction provides acceptable lattice-matched materials either has a larger bandgap as a material in the absorption region as well as good electron transport properties and increasing the rate of the electron- hole pair generation.

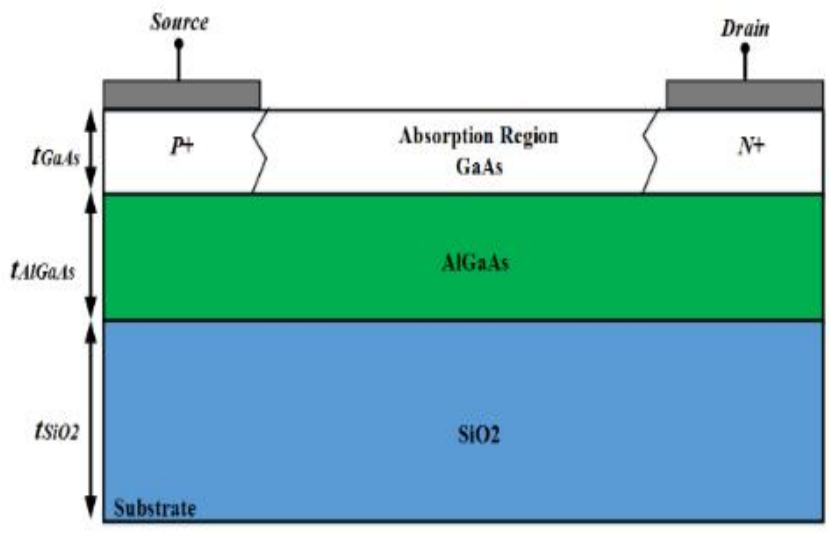

(a)

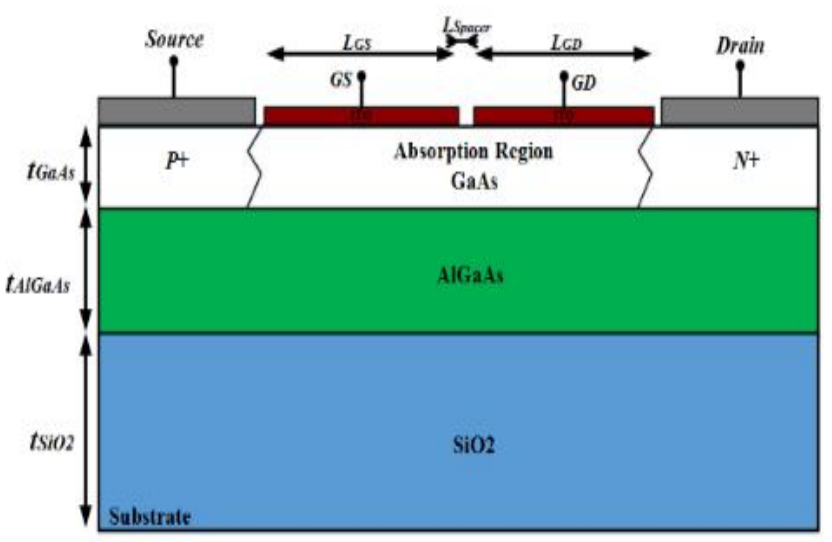

(b)

Fig. 1. (a) The regular PIN Photodiode (b) the novel Field Effect Photodiode structures.

To explain the reason for the use of Gallium arsenide as an absorption region material, it can mention to the some figure of merits from this material such as of the very high electron mobility that enables superior high frequency operation achieved and favor for using in the fast optical devices such GaAs lasers, Light Emitting Diode, Photodetectors, and Optical switches. Below the $\mathrm{n}-\mathrm{GaAs}$ layer, we have set a layer of AlGaAs to ensure the separation of the hetero-junction interface from the doped GaAs region [17]. This is critical if high electron mobility is to be achieved. To provide the lower parasitic capacitance, we have set $\mathrm{SiO}_{2}$ as a substrate. The structures of the regular PIN photodiode and the proposed devices have illustrated in Fig. 1(a) and (b).

To design the proposed, we have used two semiconductors, $\mathrm{GaAs}$ and $\mathrm{AlGaAs}$, because these semiconductors have a direct energy bandgap and do not require phonon interactions (crystal lattice vibrations) for the necessary momentum to absorb photons. The reason for using the hetero-junction $\mathrm{GaAs} / \mathrm{AlGaAs}$ was to have the highest lattice matching between the two materials. The substrate layer is made of $\mathrm{SiO}_{2}$. The doping densities of the drain and source areas are $\mathrm{n}^{+}$and $\mathrm{p}^{+}$with a density of $10^{19} \mathrm{~cm}^{-3}$, respectively, and the absorption region, which is the same as the depletion region, has n-type doping with a lightly doping concentration or intrinsic with a density of $10^{14} \mathrm{~cm}^{-3}$.

Two transparent indium tin oxide Schottky gate electrodes (ITO) are mounted over the absorption region. In choosing this contact, it has been taken into account that the Schottky contact has the lowest contact with its substrate and thus increases the response speed of the device in optical applications and fast switching.

The adjacent gate electrode to the drain contact is a GateDrain (GD), and the adjacent gate electrode to the source contact is a Gate-Source (GS), respectively. Two gates are in the same length $\mathrm{L}_{\mathrm{GD}}=\mathrm{L}_{\mathrm{Gs}}$ and are used to control the density of the carriers in the absorption region by changing their biases and polarities. For having the proper performance, both the gates must be located near together for the FEPD. In this paper, we have chosen the FEPD structure like a symmetrical structure, meaning that the source and drain lengths can have an equal length $L_{D}=L_{S}$.

\section{FEPD Simulations and Operation Modes}

To simulate and extract the FEPD parameters, we have used TCAD tools as a semiconductor drift-diffusion solver. Models include Shockley-Read-Hall (SRH), Auger (AUGER), the dependence of the carrier mobility on the doping concentration, Lombardi mobility model (CVT), Bandgap narrowing, Fermi statistic dependence (FERMIDIRAC), and Lateral electric field-dependent mobility.

\section{$3.1 \quad$ ON-state}

By illuminating the absorption region with a visible light that has photon energy greater than of n-GaAs layer bandgap on top of the FEPD, the absorbed photons transfer energy to the atoms of the radiated $\mathrm{n}-\mathrm{GaAs}$ layer, moving these holes and electrons carriers to their conduction bands. Once there, the individual carrier may or may not contribute to the current flow [18]. Carriers are released within the absorption region and producing electron-hole pairs (EHP). By applying $\mathrm{V}_{\mathrm{D}}>0$ while the source is grounded, the FEPD is in the reverse bias. In this case, the width of the depletion region increases, and the electric field causes more electrons transported to the drain contact and more holes transported to the source contact.

In the first mode, by biasing the $\mathrm{V}_{\mathrm{GS}}<0$ while $\mathrm{V}_{\mathrm{GD}}>0$, more generated holes accumulated under the GS contact, and more generated electrons accumulated under the GD contact. Here we have a $p+-p-n-n+$ structure and the FEPD works in its $O N$ state. Hence the magnitude of the output current versus optical wavelength of the FEPD in its $\mathrm{ON}$-state is several orders of magnitude higher than in comparison with the regular PIN-PD, as shown in Fig. 2. 


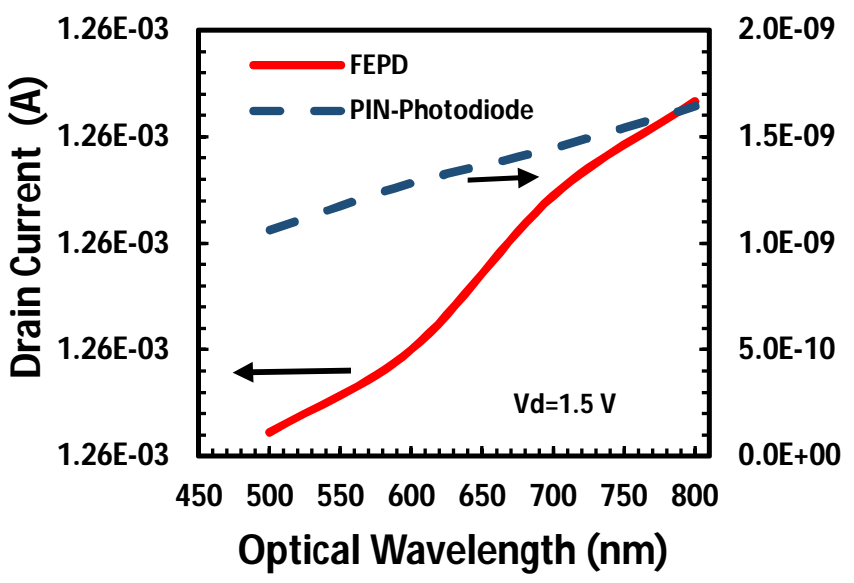

Fig. 2. Output Current versus Optical Wavelength of the FEPD in ON-state and the regular PIN-PD.

The collected charges at these contacts produce an electric signal in the external circuit. Fig. 3 (a), (b) illustrate the energy band diagrams of both the regular PIN-PD and FEPD in its ONstate.

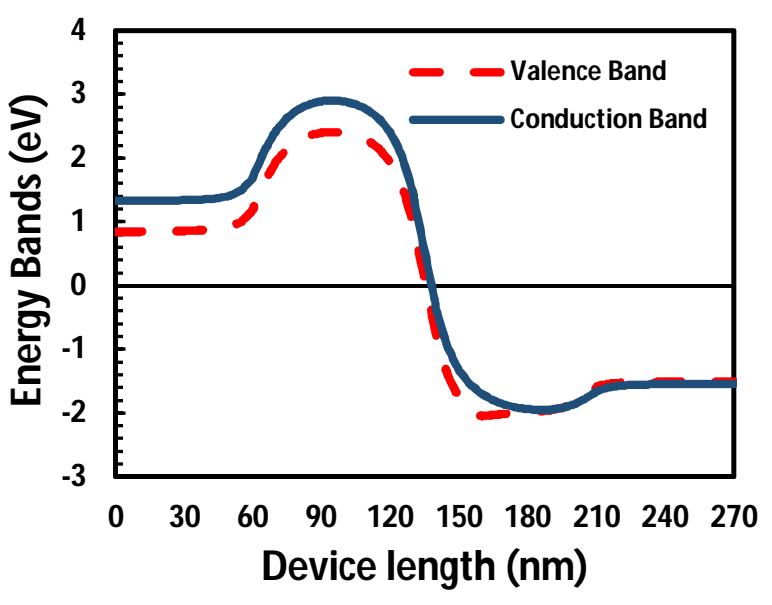

(a)

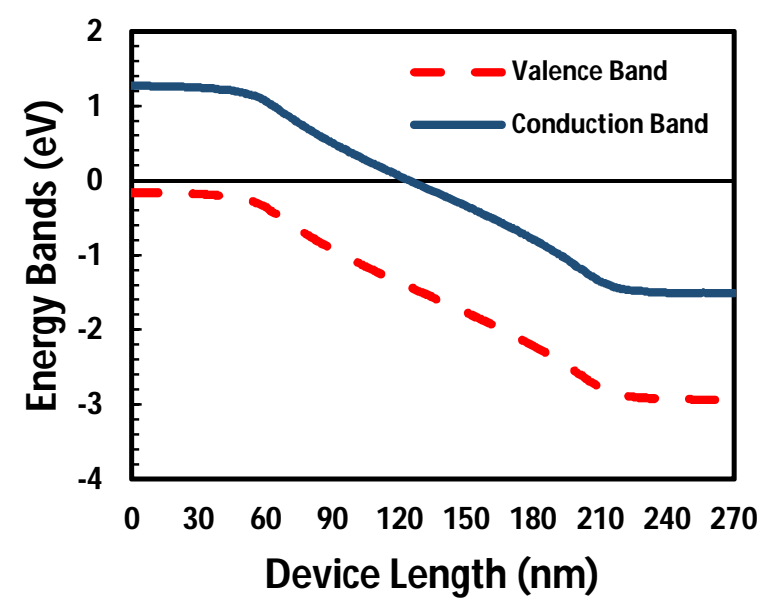

(b)

Fig. 3. Energy Band Diagrams for both the regular PIN-PD and novel FEPD in ON-state. Curves are extracted along a cutline from drain to source at the center of n-GaAs film. (a) novel FEPD, (b) regular PIN-PD.

\subsection{OFF- state}

In the second mode, by biasing the $\mathrm{V}_{\mathrm{GS}}>0, \mathrm{~V}_{\mathrm{GD}}<0, \mathrm{~V}_{\mathrm{D}}>0$ while the source remains grounded, the width of the depletion region increases due to reverse bias, and the electric field cause more electrons transported to the drain contact and more holes transported to the source contact, but here regarding to the gates bias; more generated holes accumulated under the $\mathrm{G}_{\mathrm{D}}$ contact and more generated electrons accumulated under the GS contact, here we have a $p+-n-p-n+$ structure and the FEPD works in its $O F F$-state that results to considerably decreasing the output current magnitude in the defined wavelength interval. Fig. 4 has shown the energy band diagrams of the FEPD in its OFF-state.

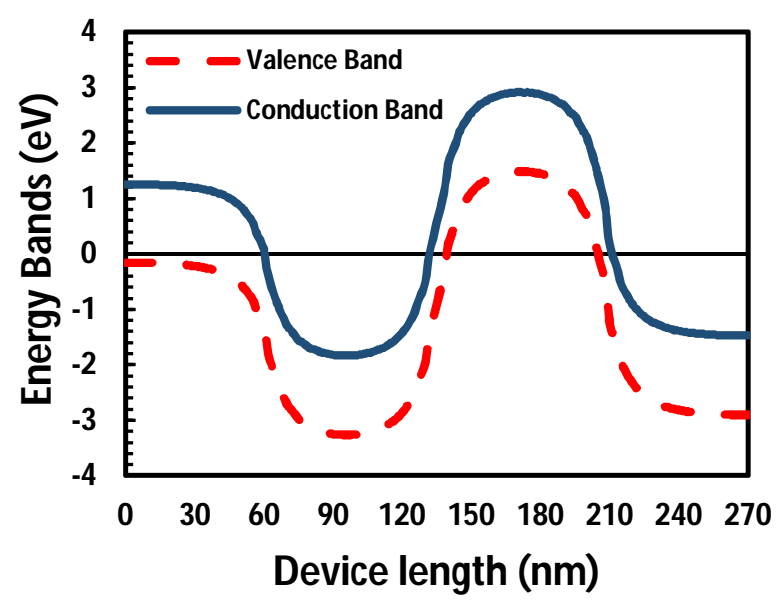

Fig. 4. Energy band diagrams for the novel FEPD in OFF-state. Curves are extracted along a cutline at the center of n-GaAs film.

Figures 5 and 6 illustrate the carrier densities versus device length of the FEPD in both the ON and OFF states, respectively.

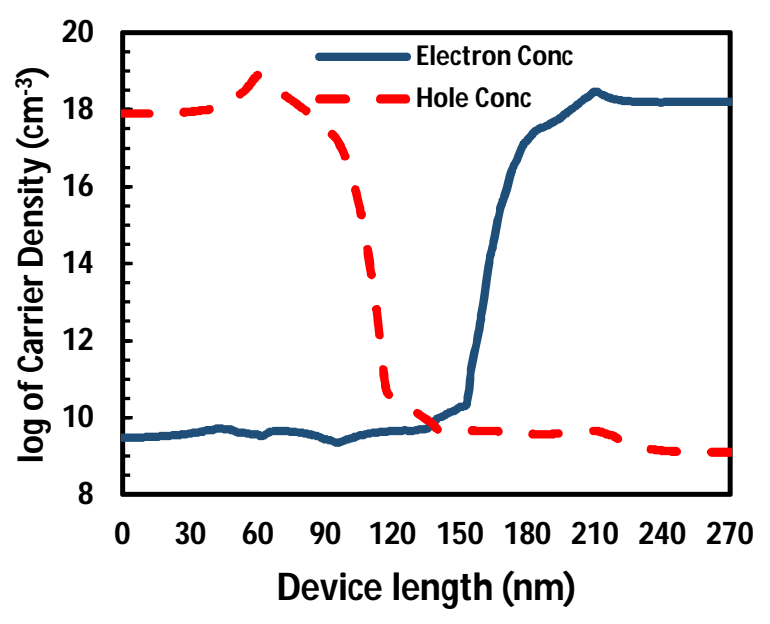

Fig. 5. Carrier density of the novel FEPD. Curves are extracted along a cutline at the center of $n-G a A s$ film in the ON-state. 


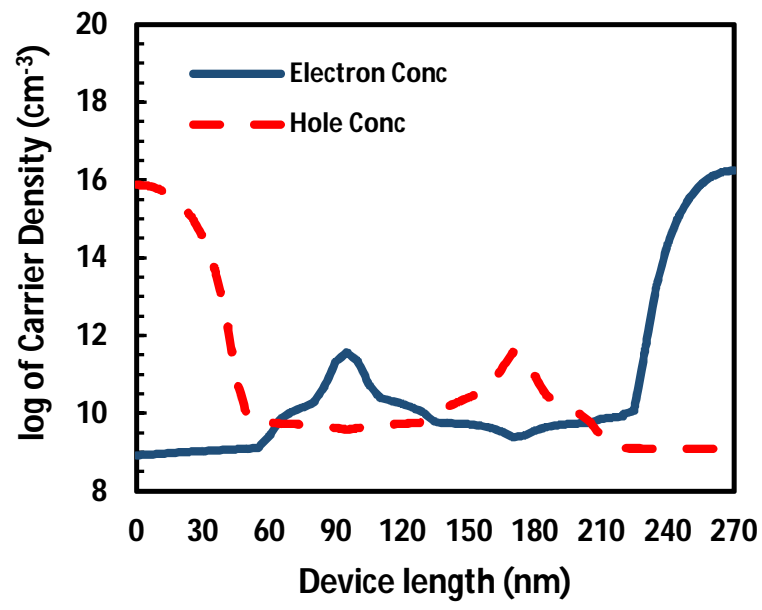

Fig. 6. Carrier density of the novel FEPD. Curves are extracted along a cutline at the center of $n-G a A s$ film in the OFF-state.

According to the simulation results adopted from Fig. 5 (a), the heavy doped-regions in the source/drain of the novel FEPD change the potential distribution, and regarding the gates bias in the first mode $\left(\mathrm{V}_{\mathrm{GS}}<0\right.$ and $\left.\mathrm{V}_{\mathrm{GD}}>0\right)$, we have a $p+-p-n-n+$ structure and the FEPD works in its ON-state. By changing the gates bias polarity in the second mode $\left(\mathrm{V}_{\mathrm{GS}}>0\right.$ and $\left.\mathrm{V}_{\mathrm{GD}}<0\right)$. Two gates induct much higher potential barrier in the absorption region. Hence, a large temporary reversed-junction and perfect $p+-n-p-n+$ structure have formed inside the novel FEPD as illustrated in Fig. 5 b.

The output current versus optical wavelength in the visible range results of simulating the FEPD in the OFF-state has been demonstrated in Fig. 7.

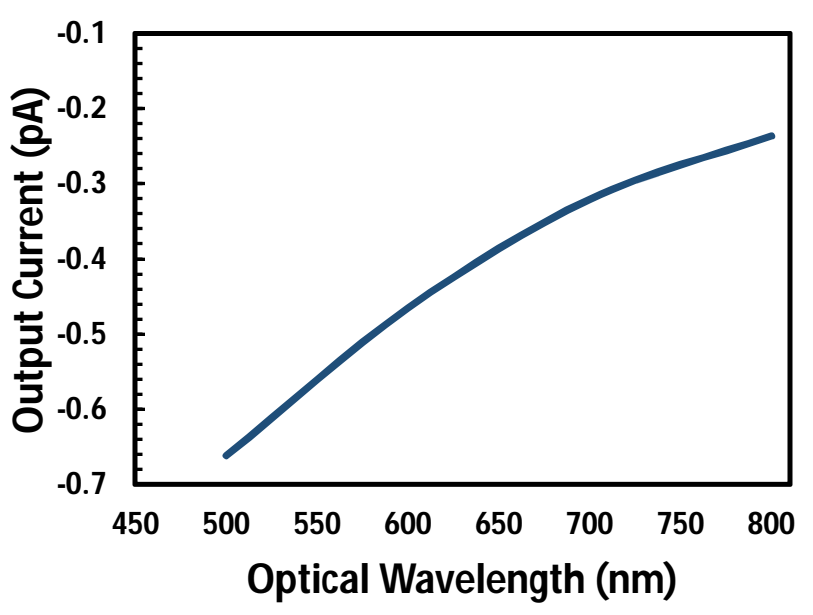

Fig. 7. Output current versus optical wavelength of the FEPD in the OFF-state.

\section{$3.2 I_{\text {ON }} / I_{\text {OFF }}$ ratio}

After calculating the ION and $\mathrm{I}_{\mathrm{OFF}}$ in both ON and OFF modes, we have obtained the main parameter of the proposed device, which has an $I_{O N} / I_{O F F}$ ratio and plays a crucial role in the fast optical switching applications, as shown in Fig.8.

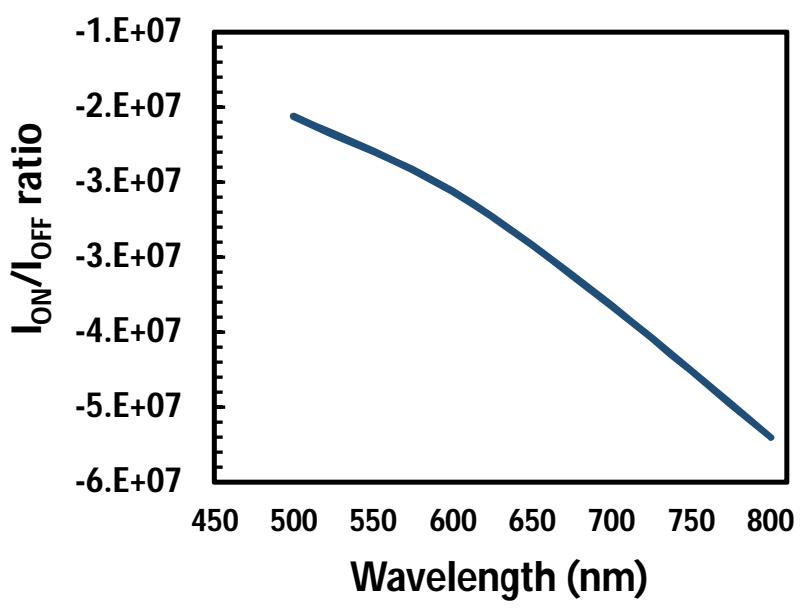

Fig. 8. $\mathrm{I}_{\mathrm{ON}} / \mathrm{I}_{\mathrm{OFF}}$ ratio versus optical wavelength for the novel FEPD.

Table 1 shows the various structures according to gates polarity and work modes for FEPD versus optical wavelength.

Table 1.

Different work modes of novel FEPD versus wavelength at $\mathrm{V}_{\mathrm{DS}}>0$

\begin{tabular}{lcc}
\hline \hline Mode & Gate Biases & Structure \\
\hline ON & $\mathrm{V}_{\mathrm{GD}}=+3 \mathrm{~V}, \mathrm{~V}_{\mathrm{GS}}=-3 \mathrm{~V}$ & $p^{+}-p-n-n^{+}$ \\
OFF & $\mathrm{V}_{\mathrm{GD}}=-3 \mathrm{~V}, \mathrm{~V}_{\mathrm{GS}}=+3 \mathrm{~V}$ & $p^{+}-n-p-n^{+}$ \\
\hline \hline
\end{tabular}

\subsection{Dark current}

Dark current is a sum of the various phenomena such as non-radiative currents generated by SRH recombination and band-to-band tunneling current. In some references, the dark current is considered as a leakage current in the absence of light. Fig. 9 shows the value of the dark current relative to the drain voltage for the gate biases, $\mathrm{V}_{\mathrm{GS}}=+3 \mathrm{~V}$ and $\mathrm{V}_{\mathrm{GD}}=-3 \mathrm{~V}$, in the off state and without the presence of radiation.

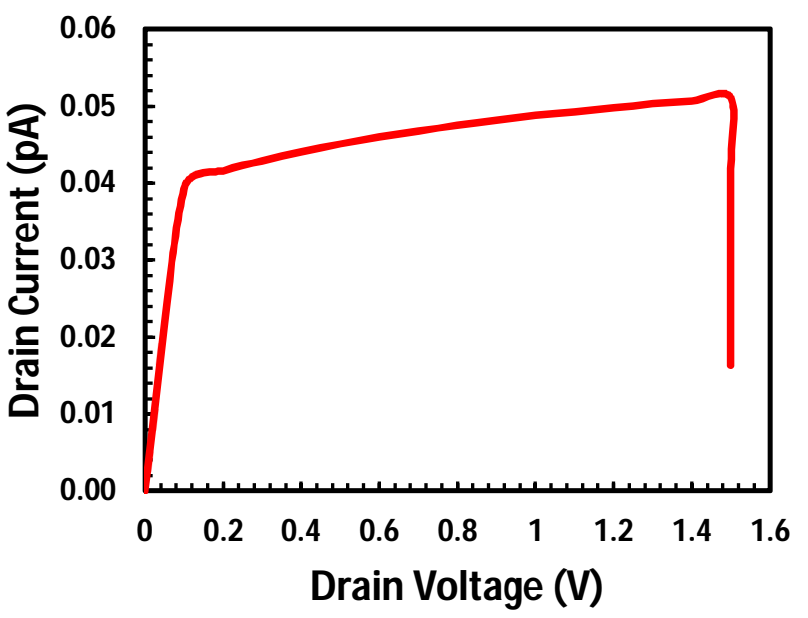

Fig. 9. Dark current versus the drain voltage in the OFF state. 


\subsection{Spectral response in on and off modes}

To better understand the concept of sensitivity of a photodiode, it is necessary to introduce a parameter called the spectral response, which is the change of the output signal as a function of the changes in the wavelength of the input signal. We have demonstrated this parameter according to the wavelength range of the absorbed photon and considering the different applied biases to the gates installed on the absorption region. The spectral response of the device in the OFF state of the device has shown in Fig. 10 at the wavelength range from $\lambda$ $=300 \mathrm{~nm}$ to $\lambda=800 \mathrm{~nm}$.

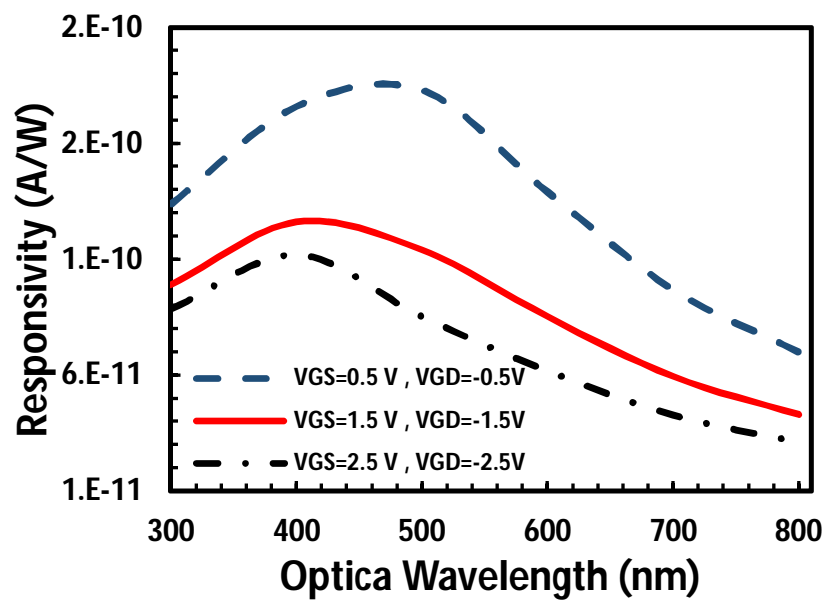

Fig.10. Spectral response of the device in the OFF state in different gates biases.

Fig. 11 shows the spectral response of the device in the $\mathrm{ON}$ state at gates biases $\mathrm{V}_{\mathrm{GS}}=-3 \mathrm{~V}$ and $\mathrm{V}_{\mathrm{GD}}=+3 \mathrm{~V}$ at the wavelengths from $\lambda=300 \mathrm{~nm}$ to $\lambda=800 \mathrm{~nm}$.

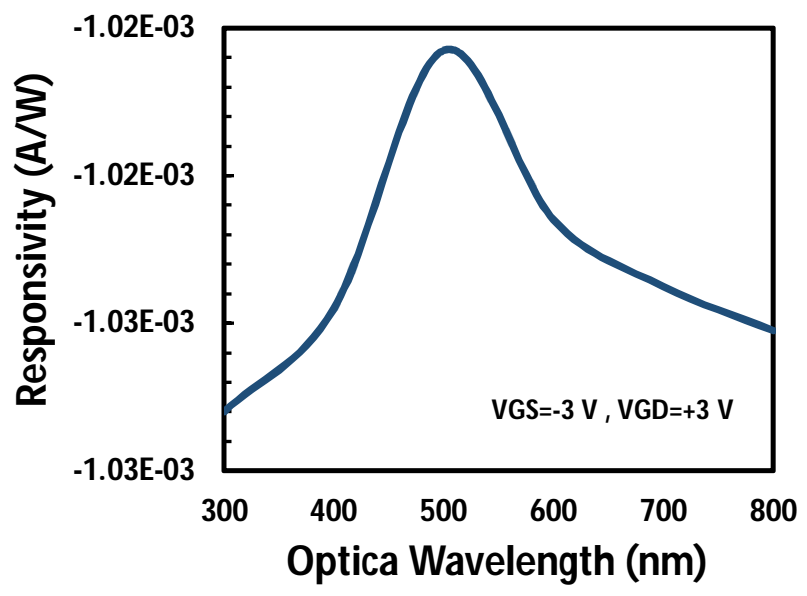

Fig. 11. Spectral response of the device in the ON state.

According to the magnitude of the spectral response in the ON-state and the wavelength range from $\lambda=300 \mathrm{~nm}$ to $\lambda=800$ $\mathrm{nm}$, it is observed that the device in the range below the wavelength of $\lambda=520 \mathrm{~nm}$, has shown good response.
The structure details and simulation results for the novel FEPD in ON and OFF states have demonstrated in Table 2.

TABLE II

Simulation results and structure details for novel FEPD in ON and OFF states.

\begin{tabular}{ll}
\hline \hline Quantity & Value \\
\hline$I_{\text {ON }}(A)$ & $1.25 \times 10^{-3}$ \\
$I_{\text {OFF }}(A)$ & $-2.37 \times 10^{-11}$ \\
$I_{O N} / I_{O F F}$ & $-5.31 \times 10^{7}$ \\
Light intensity $\left(W / \mathrm{cm}^{3}\right)$ & 2.6 \\
Wavelength $(\mu \mathrm{m})$ & $0.3<\lambda<0.8$ \\
Source doping & $\mathrm{n}^{+}=1 \times 10^{19} \mathrm{~cm}^{-3}$ \\
Drain doping & $\mathrm{p}^{+}=1 \times 10^{19} \mathrm{~cm}^{-3}$ \\
Absorption Region & $1 \times 10^{14} \mathrm{~cm}^{-3}$ \\
Thickness of $n$-GaAs film $\left(t_{\text {GaAs }}\right)$ & $20 \mathrm{~nm}$ \\
Thickness of AlGaAs film $\left(t_{\text {AlGaAs }}\right)$ & $80 \mathrm{~nm}$ \\
Thickness of Substrate Sio2 $\left(t_{\text {Sio } 2}\right)$ & $200 \mathrm{~nm}$ \\
Total length & $270 \mathrm{~nm}$ \\
Internal Quantum Efficiency & $73 \%$ \\
\hline \hline
\end{tabular}

\section{Conclusions}

To control output current and fast optical switching of a regular PIN photodiode (PIN-PD), a novel device named Field Effect Photodiode (FEPD) was introduced in this paper. The proposed device can be fabricated by the standard technology of the regular PIN photodiode and conventional MESFET structures despite two indium tin oxide (ITO) Schottky contact gates over the absorption region. For controlling an output current either properly turning the device off in the visible wavelength interval. The top layer is an $\mathrm{n}-\mathrm{GaAs}$ that has an $\mathrm{Eg}=1.44 \mathrm{eV}$. Below the $\mathrm{n}-\mathrm{GaAs}$ layer, we have set a layer of AlGaAs. The GaAs/AlGaAs hetero-junction can provide acceptable lattice-matched materials regarding the larger bandgap for controlling the absorption in the structure as well as good electron transport properties. The simulation with TCAD tools software has been performed to obtain the output parameters in different bias and optical wavelength conditions. The I-V characteristics such as output current versus wavelength, carrier density, Internal Quantum Efficiency, energy band diagrams, I I and $\mathrm{I}_{\mathrm{OFF}}, I_{O N} / I_{O F F}$ ratio, dark current, and spectral response for the proposed FEPD and the regular PIN-PD have extracted. The measured characteristics were compared and the results shown graphically, then the novel FEPD exhibited good results to control and turning off the device output current. According to the results of simulating, to improve the output parameters; suggestions are provided below:

1) In order to increase the efficiency of the device, which is directly related to the ability to receive incident photons on the surface of the absorption region, it is suggested to use an antireflection layer on the surface of the absorption region to receive maximum incident light power and prevent reflection.

2) One of the problems of all electronic and optoelectronic semiconductor devices is the noise caused in the process of mobility and transfer of electrons inside the material or passing from one material to another, which has its effect as heat or 
decreasing in the linear response of the device. For this device, it is suggested to use an area next to the absorption region as an augmentation or multiplication region, such as avalanche photodiode structures, which have an internal gain based on impact ionization.

3) Proposing to change the molar fraction of binary and ternary semiconductors used in the structure of the device and to obtain the best combination for designing the absorption region that is directly related to the molar mass of the semiconductor and can be an effective parameter. In the amount of absorption and reduction of undesirable quantum effects is the light absorbing layer.

Therefore, the proposed device can be used to design in the new generations of the optical digital and analogue detectors and control an output current.

\section{References}

[1] S. L. Chuang, "Physics of Optoelectronic Devices", John Wiley \& Sons, New York, 1995.

[2] Mahmoud Shaban, Abdelrahman Zkria, Tsuyoshi Yoshitake. "Characterization and design optimization of heterojunction photodiodes comprising n-type ultra-nano-crystalline diamond/hydrogenated amorphous carbon composite and p-type Si" Materials Science in Semiconductor Processing. $86 \quad$ (2018) 115-121. https://doi.org/10.1016/j.mssp.2018.06.028.

[3] P. Bhattacharya, "Semiconductor Optoelectronic Devices", 2nd. ed., Prentice Hall, Upper Saddle River. NJ, 1997.

[4] Jie Xu a, Xiupu Zhang a, Ahmed Kishk, "Design of high speed InGaAs/InP one-sided junction photodiodes with low junction capacitance", Optics Communications 437 (2019) 321-329. https://doi.org/ 10.1016/j. optcom. 2018.12.085.

[5] Jhih-Min Wun, Yu-Wen Wang, and Jin-Wei Shi,"Ultrafast Uni-Traveling Carrier Photodiodes with GaAs0.5Sb0.5/In0.53Ga0.47As Type-II Hybrid Absorbers for High-Power Operation at THz Frequencies", IEEE JOURNAL OF SELECTED TOPICS IN QUANTUM ELECTRONICS. VOL. $24, \quad$ NO. 2, MARCH/APRIL 2018 http://dx.doi. org/ 10.1109/JSTQE.2017.2741106

[6] H.J. Song, T. Nagatsuma, "Present and Future of Terahertz Communications", IEEE Trans. Terahertz Sci. Technol. 1 (2011) 256263, http://dx.doi.org/10.1109/ THZ.2011.2159552.

[7] A.J. Seeds, K.J. Williams. Microwave Photonics, J. Lightwave Technol.24 (2006) 4628-4641, http://dx.doi.org/10.1109/JLT.2006 .885787.

[8] G. Chattopadhyay. Technology, capabilities, and performance of low power terahertz sources. IEEE Trans. Terahertz Sci. Technol. 1 (2011) 33-53, http://dx.doi. org/10.1109/TTHZ.2011.2159561.

[9] H. Ito, S. Kodama, Y. Muramoto, T. Furuta, T. Nagatsuma, T. Ishibashi, "High-speed and high-output InP-InGaAs unitraveling-carrier photodiodes", IEEE J. Sel. Top. Quantum Electron. 10 (2004) 709-727, http://dx.doi.org/10.1109/JSTQE.2004.833883.

[10] H. Ito, T. Furuta, Y. Hirota, T. Ishibashi, A. Hirata, T. Nagatsuma, H. Matsuo, T. Noguchi, M. Ishiguro, "Photonic millimetre-wave emission at $300 \mathrm{GHz}$ using an antenna-integrated uni-travelling-carrier photodiode", Electron. Lett. 38(2002)989-990, http://dx.doi.org/10.1049/el: 20020667.

[11] R. S. Tucker, A. J. Taylor, C.A. Burrus, G. Eisenstein, J. M. Wiesenfeld, "Coaxially mounted $67 \mathrm{GHz}$ bandwidth InGaAs PIN photodiode", Electron. Lett. 22(1986)917-918, http://dx.doi.org/10.1049/el: 19860625.

[12] Iraj Sheikhian, Foad Sharafi," Improved nanoscale field effect diode", IET Circuits Devices Syst., 2019, Vol. 13 Iss. 4, pp. 544-547. https://doi.org/10.1049/iet-cds.2018.5138.

[13] Raissi, F., Sheikhian, I,'Nanoscale transistor device with large current handling capability", European Patent EP1965437A1, 2008.

[14] Sheikhian, I., Raissi, F, "Simulation results for nanoscale field effect diode", IEEE Trans. Electron. Devices, 2007, 54, (3), pp. 613-617. http://dx.doi: 10.1109/TED.2006.890600.

[15] Sheikhian, I., Raissi, F, "High-speed digital family using field effect diode", IET Electron. Lett. 2003, 39, (4), pp. 345-347. http://dx.doi: 10.1049/el: 20030251.
[16] Y. Sun, J. C. Campbell, S. Wang, A. L. Beck, A. Chen, A. Yulius, and J. M. Woodall, "Drift-Dominated InP Photodetectors with High Quantum Efficiency", Proc. Intl. Conf. on Indium Phosphide and Related Materials. 2003, pp. 502-505. http://dx.doi: 10.1109/ICIPRM.2003.1205426.

[17] Nadir Dagli, "High-Speed Photonic Devices (Series in Optics and Optoelectronics)", Taylor \& Francis (2003).

[18] Jerald Graeme, "Photodiode amplifiers op-amp solutions", McGraw Hill (1996). 